\title{
Análisis del cumplimiento de Garantía de Oportunidad en seis patologías Auge en un consultorio rural
}

\author{
NATALIA URQUIOLA ${ }^{(1)}$ y MARIA JOSÉ ZAMORANO ${ }^{(1)}$
}

\section{RESUMEN}

El plan Auge corresponde a un sistema integral de salud que considera Acceso Universaly Garantias Explícitas asociadas a la atención de problemas prioritarios de salud. Dentro de éstas, la Garantía de Oportunidad establece plazos máximos para el diagnóstico y tratamiento de 56 patologías.

El objetivo es evaluar el cumplimiento de la garantía de oportunidad para seis patologías incluidas en el Auge, en un consultorio rural de Chile, entre mayo 2006 y marzo 2008.

Fueron evaluadas 209 interconsultas (IC) emitidas entre mayo 2006 y marzo 2008 para 6 patologías: colelitiasis, cáncer gástrico, parto prematuro, vicios de refracción, depresión y artrosis de cadera. Se estableció el cumplimiento de la garantía de oportunidad tomando como fecha de corte el 30 de marzo del 2008.

El porcentaje de cumplimiento de la Garantía de Oportunidad fue de 76,92\% para parto prematuro; $75,8 \%$ en colelitiasis; $48,21 \%$ en vicios de refracción; $36,3 \%$ en cáncer gástrico; $33,3 \%$ en artrosis de cadera y 21,82\% en depresión.

En nuestro trabajo el porcentaje de cumplimiento de la garantía de oportunidad fue menor al $80 \%$ para cada una de las patologías analizadas, con un retraso de 50,23\% (105 de 209 pacientes) en los plazos establecidos por la ley Auge.

Palabras clave: Auge, Garantía de Oportunidad, fecha de derivación, fecha de citación.

\section{ABSTRACT}

\section{ANALYSIS OF ACCOMPLISHMENT OF THE OPPORTUNITY GUARANTEE FOR SIX AUGE} PATHOLOGIES AT AN OUTPATIENT RURAL PRACTICE

The Auge regime is a comprehensive health system that considers Universal Access and Explicit Guarantees with regard to the care of priority health issues. Among these, the Opportunity Guarantee establishes maximum deadlines for the diagnosis and treatment of 56 pathologies.

The purpose is to assess the accomplishment of the Opportunity Guarantee for six pathologies included in the Auge regime, at an outpatient rural practice in Chile, between May 2006 and March 2008 .

A total of 209 consultation referrals issued between May 2006 and March 2008 for 6 pathologies were assessed: cholelithiasis, gastric cancer, premature labor, refractive pathologies, depression and hip joint arthrosis. Accomplishment of the Opportunity Guarantee was defined using May 30 2008 as cutoff date.

The percentage of accomplishment of the Opportunity Guarantee was $76.92 \%$ for premature labor; $75.8 \%$ for cholelithiasis; $48.21 \%$ for refractive pathologies; $36.3 \%$ for gastric cancer; $33.3 \%$ for hip arthrosis and $21.82 \%$ for depression.

(1) Internas Séptimo año de Medicina. Universidad de Chile. Campus Centro. Pje. Dagoberto Godoy 182. Villa Luis Gumucio. San Joaquín. Santiago.Chile.naty.urqui@gmail.com 
In the present work the percentage of accomplishment of the opportunity guarantee was below 80\% for each of the pathologies assessed, with a delay in compliance with the deadlines set by the Auge Act affecting 50.23\% of the cases (105 of 209 patients).

Keywords: Auge, Opportunity guarantee, referral date, appointment date.

\section{INTRODUCCIÓN}

La salud es un bien social y el acceso a ella constituye un derecho esencial de las personas. Chile ha presentado grandes cambios durante esta década en el ámbito de la salud. Uno de los más significativos en este sentido ha sido la implementación de un régimen de garantías en salud, el Auge, instrumento de regulación sanitaria que considera acceso universal para prestaciones integrales y Garantías Explícitas asociadas a la atención de prioridades ${ }^{1}$.

El Auge tiene como fin priorizar racionalmente el uso de los recursos, dirigiéndolos hacia aquellos problemas que más afectan a la población, estableciendo garantías de acceso, oportunidad, calidad y protección financiera. Dentro de éstas, la garantía de oportunidad establece que los personas tendrán la seguridad de ser atendidas en un plazo máximo. De no cumplirse, podrán reclamar a la Superintendencia de Salud, que los enviará a un centro hospitalario donde efectivamente puedan ser atendidos, asumiendo los costos la Isapre o Fonasa, según corresponda ${ }^{2}$.

La puesta en marcha del Auge comenzó en agosto del 2002, cuando se explicitan garantías para tres problemas de salud. Actualmente son 56 las patologías incluidas, todas con sus garantías respectivas ${ }^{3}$.

El presente trabajo tiene como propósito evaluar el cumplimiento de la garantía de oportunidad para seis patologías incluidas en el Auge, en un consultorio rural de Chile, entre mayo 2006 y marzo 2008.

\section{MATERIAL Y MÉTODO}

El presente trabajo corresponde a un estudio descriptivo realizado en el Consultorio Quinta de Tilcoco, ubicado en la VI Región, Chile.
Entre las patologías incluidas en el Auge y atendidas en el consultorio, se seleccionaron seis para su análisis:

1. Prevención del parto prematuro en embarazadas con factores de riesgo.

2. Colecistectomía preventiva del cáncer de vesícula en personas de 35 a 49 años.

3. Cáncer gástrico.

4. Vicios de refracción en personas de 65 años y más.

5. Artrosis de cadera leve o moderada en personas de 55 años y más.

6. Depresión severa en personas de 15 años y más.

Las garantías de oportunidad para cada una de estas patologías son:

- Parto prematuro: consulta con especialista dentro de 10 días desde la derivación ${ }^{4}$.

- Colecistectomía preventiva: diagnóstico con ecotomografía abdominal dentro de 60 días y cirugía dentro de 90 días de confirmado el diagnóstico de cálculos en la vesícula y/o vías biliares ${ }^{5}$.

- Cáncer gástrico: consulta con especialista dentro de 45 días desde la sospecha ${ }^{6}$.

- Vicios de refracción: diagnóstico por especialista dentro de 180 días desde sospecha?.

- Artrosis de cadera leve o moderada: consulta con especialista dentro de 120 días desde la derivación ${ }^{8}$.

- Depresión severa: consulta con psiquiatra dentro de 30 días desde la derivación ${ }^{9}$.

Se procedió a revisar la totalidad de interconsultas (IC) realizadas por estos diagnósticos en el período comprendido entre mayo 2006 y marzo 2008. Para ello se contó con las copias y el libro de registro diario de IC por patología Auge. La información recopilada incluyó: nombre comple- 
to del paciente, edad, diagnóstico, especialidad en la cual se solicitó atención, hospital de derivación y fecha de emisión de la IC.

Se recopiló un total de 209 IC con los diagnósticos buscados, correspondiendo cada una a un paciente, repartiéndose de la siguiente manera:

- 55 IC a psiquiatría por diagnóstico de depresión severa, derivados a Hospital de Rengo.

- 12 IC a traumatología por diagnóstico de artrosis de cadera leve o moderada, derivados a Hospital de Rancagua.

- 56 IC a oftalmología por diagnóstico de vicio de refracción (miopía, astigmatismo, hipermetropía), derivados a Hospital de Rancagua.

- 11 IC a gastroenterología por diagnóstico de cáncer gástrico, derivados a Hospital de Rancagua.

- 13 IC a Poli Alto Riesgo Obstétrico (ARO) por diagnóstico de factores de riesgo para parto prematuro, derivados a Hospital de Rengo.

- 52 IC a radiología por sospecha de colelitiasis y 10 IC a cirugía con diagnóstico confirmado de colelitiasis, ambos derivados a Hospital de Rengo.

Para cada una de estos 209 pacientes se estableció si fue dada una hora en la especialidad requerida. Luego se calculó la diferencia en días desde la fecha de derivación hasta la fecha de citación con especialista. En el caso de los pacientes sin citación, se calculó la diferencia en días con respecto al 30 de marzo de 2008, que en este estudio se estableció como fecha de corte para verificar el cumplimiento de la garantía de oportunidad.

Una vez obtenida esta información, se analizó, para cada paciente, si se cumplió la garantía de oportunidad explicitada en el Auge. Se consideró para esto:

- Cumplió garantía: si el tiempo transcurrido entre la fecha de derivación y la fecha de citación fue menor o igual al plazo máximo esta- blecido en Auge para la patología.

- No cumplió garantía: si el tiempo transcurrido entre la fecha de derivación y la fecha de citación fue mayor al plazo máximo establecido en Auge para la patología.

De este modo se calculó el porcentaje de cumplimiento de la garantía de oportunidad para cada una de las seis patologías y se realizó un análisis comparativo entre ellas. Adicionalmente se calculó el porcentaje de incumplimento de la garantía para el total de IC analizadas (209 pacientes) y el intervalo promedio de días existente entre la realización de la IC y la citación con especialista.

\section{RESULTADOS}

\section{Colecistectomía preventiva}

Del total de 62 pacientes derivados con diagnóstico de colelitiasis, un 75,8\% (47 pacientes) tenía hora para especialista dentro del plazo establecido en la garantía explícita de oportunidad Auge y un 24,19\% (15 pacientes) contaba con hora fuera del plazo máximo o simplemente no tenía fecha de citación.

De los 15 pacientes con fecha de citación fuera de plazo, un 92\% llevaba más de 240 días de espera desde la fecha de derivación con especialista (Figura 1).

Figura 1. Colelitiasis. Porcentaje de pacientes según número de días de retraso en la garantía de oportunidad.

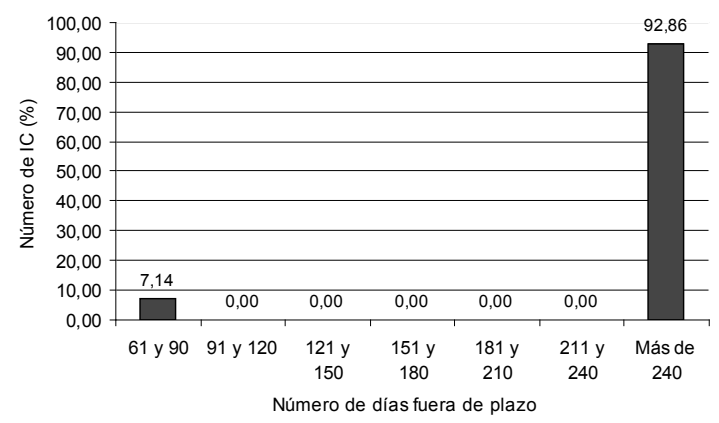




\section{Cáncer gástrico}

Del total de 11 pacientes derivados con diagnóstico de cáncer gástrico, sólo un $36,3 \%$ (cuatro pacientes) tenía hora para especialista dentro del plazo establecido en la garantía explícita de oportunidad Auge y un 63,6\% (7 pacientes) contaba con hora fuera del plazo máximo o simplemente no tenía fecha de citación.

De los 7 pacientes con fecha de citación fuera de plazo, un 29\% llevaba más de 135 días de espera desde la fecha de derivación con especialista (Figura 2).

Figura 2. Cáncer gástrico. Porcentaje de pacientes según número de días de retraso en la garantía de oportunidad.

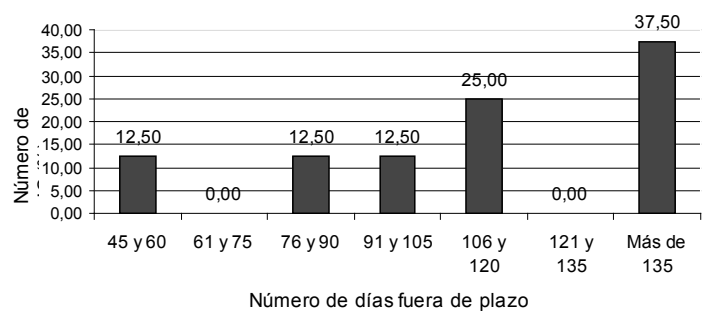

\section{Vicios de refracción}

Del total de 56 pacientes derivados con diagnóstico de vicios de refracción, un 48,21\% (27 pacientes) tenía hora para especialista dentro del plazo establecido en la garantía explícita de oportunidad Auge y un 51,78\% (29 pacientes) contaba con hora fuera del plazo máximo o simplemente no tenía fecha de citación.

De los 29 pacientes con fecha de citación fuera de plazo, un 48\% llevaba entre 211 y 240 días de espera desde la fecha de derivación con especialista y un $15 \%$ más de un año de espera (Figura 3).
Figura 3. Vicios de refracción. Porcentaje de pacientes según número de días de retraso en la garantía de oportunidad.

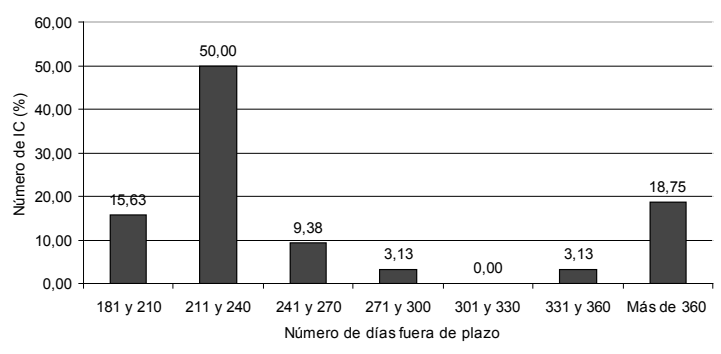

\section{Artrosis de cadera leve o moderada}

Del total de 12 pacientes derivados con diagnóstico de artrosis de cadera leve o moderada, sólo un 33,3\% (4 pacientes) tenía hora para especialista dentro del plazo establecido en la garantía explícita de oportunidad Auge y un 66,6\% (8 pacientes) contaba con hora fuera del plazo máximo o simplemente no tenía fecha de citación.

De los ocho pacientes con fecha de citación fuera de plazo, un $85 \%$ llevaba más de 300 días de espera desde la fecha de derivación (Figura 4).

Figura 4. Artrosis de cadera leve o moderada. Porcentaje de pacientes según número de días de retraso en la garantía de oportunidad.

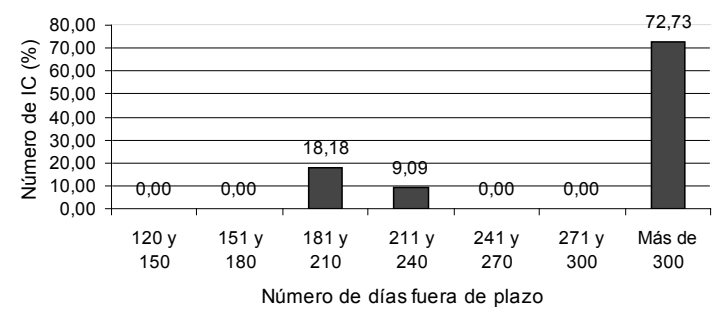




\section{Depresión severa}

Del total de 55 pacientes derivados con el diagnóstico de depresión severa, un 21,81\% (12 pacientes) tenía hora para especialista dentro del plazo establecido en la garantía explícita de oportunidad Auge y un 78,18\% (43 pacientes) contaba con hora fuera del plazo máximo o simplemente no tenía fecha de citación.

De los 43 pacientes con fecha de citación fuera de plazo, un $23 \%$ llevaba entre 31 y 60 días de espera desde la fecha de derivación y un $24 \%$ entre 90 y 120 días (Figura 5).

Figura 5. Depresión severa. Porcentaje de pacientes según número de días de retraso en la garantía de oportunidad.

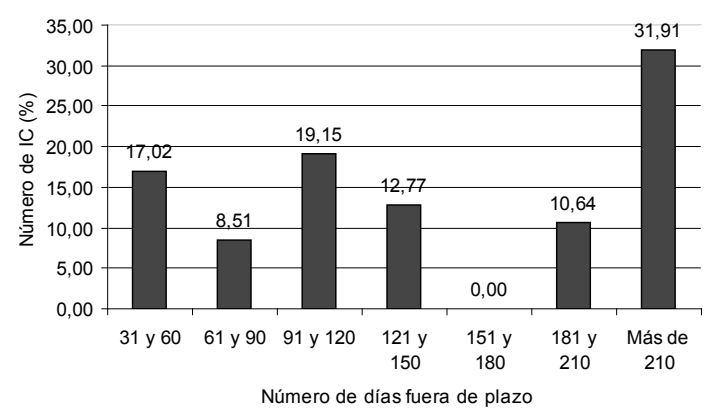

\section{Parto prematuro}

Del total de 13 pacientes derivadas con diagnóstico de factores de riesgo para parto prematuro, un $76,92 \%$ (10 pacientes) tenía hora para especialista dentro del plazo establecido en la garantía explícita de oportunidad Auge y un $23,1 \%$ (3 pacientes) contaba con hora fuera del plazo máximo o simplemente no tenía fecha de citación.
Figura 6. Comparación entre seis patologías AUGE según porcentaje de cumplimiento de Garantía de Oportunidad

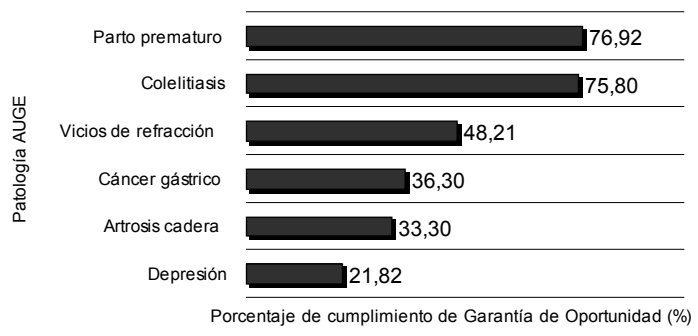

\section{DISCUSIÓN}

En nuestro trabajo el porcentaje de cumplimiento de la garantía de oportunidad fue menor al $80 \%$ para cada una de las patologías analizadas. La depresión severa presentó el menor porcentaje con un $21,82 \%$, es decir, sólo 12 de los 55 pacientes derivados con el diagnóstico contaban con citación a especialista dentro del plazo establecido por la normativa legal.

En el otro extremo se encuentra parto prematuro que presentó un $76,92 \%$ de cumplimiento de la garantía de oportunidad, con 10 de 13 pacientes que contaban con citación a especialista dentro del plazo establecido. En la misma situación se encuentra la patología colelitiasis, con un $75,8 \%$ de cumplimiento ( 47 de 62 pacientes). Lo anterior concuerda con lo informado en otro estudio, en que el porcentaje de cumplimiento de los plazos para la patología colelitiasis bordeaba el $70 \%{ }^{10}$.

En cuanto al incumplimiento de la garantía de oportunidad, éste fue de un 50,23\%, lo que corresponde a 105 de los 209 pacientes incluidos en este estudio, presentándose el mayor 
porcentaje de incumplimiento en los pacientes derivados con diagnóstico de depresión severa, alcanzando un 78,18\% (43 de 55 pacientes). Lo anterior concuerda con lo reportado en un informe realizado por el Fondo Nacional de Salud (Fonasa) entre mayo y junio 2007, en el cual se analizó el cumplimiento de los plazos de diagnóstico y tratamiento para 4 patologías Auge (cáncer gástrico, cáncer de próstata, hiperplasia prostática y cataratas) en las regiones Metropolitana y VI, observándose cifras promedio de hasta un $70,5 \%$ de incumplimiento ${ }^{11}$. Esta cifra es mayor a la reportada por el Ministerio de Salud con fecha 30 de junio 2008, en que el porcentaje de retraso fue de $5,18 \%$, correspondiendo a 299.587 garantías retrasadas en las 56 patologías que incluye actualmente el Auge (12, $^{32}$.

En nuestra serie el promedio de retraso entre la IC y la citación a especialista fue de 200 días, llegando a un año en el caso de 4 pacientes con diagnóstico de vicios de refracción y a más de 300 días en el caso de 6 pacientes con diagnóstico de artrosis de cadera leve o moderada.

Son necesarios futuros estudios en los que se evalúen los factores asociados al bajo porcentaje de cumplimiento de la garantía de oportunidad, incluyendo en ellos el análisis de los hospitales de derivación, a fin de conocer los recursos humanos reales, específicamente horas de especialidad con que cuentan para hacerse cargo de la demanda asistencial generada en los consultorios. Sería interesante también reevaluar a los pacientes que no contaban con hora para especialista y verificar si, finalmente, fueron atendidos ya sea en el hospital de derivación o en otro centro asistencial.

\section{AGRADECIMIENTOS}

A todo el personal del Consultorio Quinta de Tilcoco, en especial al Dr. Otazo quien nos incentivó a realizar este trabajo; a los encargados de la Oficina Auge quienes nos ayudaron en la recolección de datos y a la directora del Consultorio Quinta de Tilcoco, Sra. Sandra Fabjanovich, quien nos facilitó los recursos necesarios para llevar a cabo este estudio.

\section{REFERENCIAS}

1. Qué es el Auge. En Gobierno de Chile (en línea). Disponible en http://www.gobiernodechile.cl/ plan_auge/que_es_auge.asp

2. En qué consisten las Garantías AUGE. En Gobierno de Chile (en línea). Disponible en http:// www.gobiernodechile.cl/plan_auge/garantias. asp

3. Qué garantiza el AUGE 56. En Gobierno de Chile (en línea). Disponible en http://www.gobiernodechile.cl/plan_auge/que_garantiza.asp

4. Prevención del Parto Prematuro. En Gobierno de Chile (en línea). Disponible en http://www. gobiernodechile.cl/plan_auge/que_garantiza19. asp

5. Colecistectomía preventiva del cáncer de vesícula en personas de 35 a 49 años. En Gobierno de Chile (en línea). Disponible en http://www. gobiernodechile.cl/plan_auge/que_garantiza31. asp

6. Cáncer gástrico en personas de 15 años y más. En Gobierno de Chile (en línea). Disponible en http://www.gobiernodechile.cl/plan_auge/que_ garantiza32.asp

7. Vicios de refracción en personas de 65 años y más. En Gobierno de Chile (en línea). Disponible en http://www.gobiernodechile.cl/plan_auge/ 
que_garantiza36.asp

8. Artrosis de cadera y/o rodilla leve o moderada en personas de 55 años y más. En Ministerio de Salud Chile (en línea). Disponible en http://www. redsalud.gov.cl/gesauge/ges articu2.html

9. Depresión en personas de 15 años y más. En Gobierno de Chile (en línea). Disponible en http:// www.gobiernodechile.cl/plan_auge/que_garantiza38.asp

10. Informe Final Consolidado del Programa Nacional de fiscalización de las Garantías Explícitas en Salud. En Contraloría General de la República (en línea). Disponible en http://www. contraloria.cl/NewPortal/portal2/ShowProperty/ BEA\%20Repository/portalCGR/Documentos/ Informes_de_Auditoria/DIVISION_AUDITORIA_ADMINISTRATIVA/AREA_SALUD_AGRICULTURA_MEDIO_AMBIENTE/
AUD_SAM12_INFORME_FINAL_CONSOLIDADO_GARANTIAS_EXPLICITAS_DE_SALUD-AGOSTO_2007

11. Informe AUGE Región Metropolitana y VI región, FONASA, agosto 2007. En Colegio Médico de Chile (en línea). Disponible en http://www. colegiomedico.cl/Noticias/NoticiasDestacadas/ AnterioresAgostode2007/tabid/666/Default. aspx

12. BARRÍA M. AUGE 2005-2008 Implementación de Garantías Explícitas en Salud. En Gobierno de Chile (en línea). Disponible en http://www. supersalud.cl/568/articles-4325_recurso_1.pdf

Recepción: 29 de septiembre de 2008 Aprobación: 12 de diciembre de 2008

Usted puede comentar éste y otros artículos publicados en la Revista Chilena de Salud Pública, enviando un correo electrónico a revistasp@med.uchile.cl 